International Scientific Colloquium

Modelling for Electromagnetic Processing

Hannover, October 27-29, 2008

\title{
Vacuum Arc Remelting Time Dependent Modelling
}

\author{
V.Bojarevics, G. Djambazov and K.Pericleous
}

Vacuum arc remelting (VAR) aims at production of high quality, segregation-free alloys. The quality of the produced ingots depends on the operating conditions which could be monitored and analyzed using numerical modelling. The remelting process uniformity is controlled by critical medium scale time variations of the order 1-100 s, which are physically initiated by the droplet detachment and the large scale arc motion at the top of liquid pool [1,2].

The newly developed numerical modelling tools are addressing the 3-dimensional magnetohydrodynamic and thermal behaviour in the liquid zone and the adjacent ingot, electrode and crucible. The finite volume multiphysics modelling software PHYSICA [3] (see a description and an application in [4]) is used for the 3-dimensional thermal-fluid dynamic fields weakly coupled to the electromagnetic field. The 3-dimensional force distribution depends on the local arc position, while the arc is moving in a prescribed (given) pattern. An example of the transient simulation snapshot at a fixed time moment is shown in Figure 1. The model accounts for the buoyancy effects and the solidification front variation resulting from the arc motion. The corresponding flow in the pool and the melt front position, determined via the liquid fraction function, is demonstrated in the Figure 2 for the same time moment as in the Figure 1.
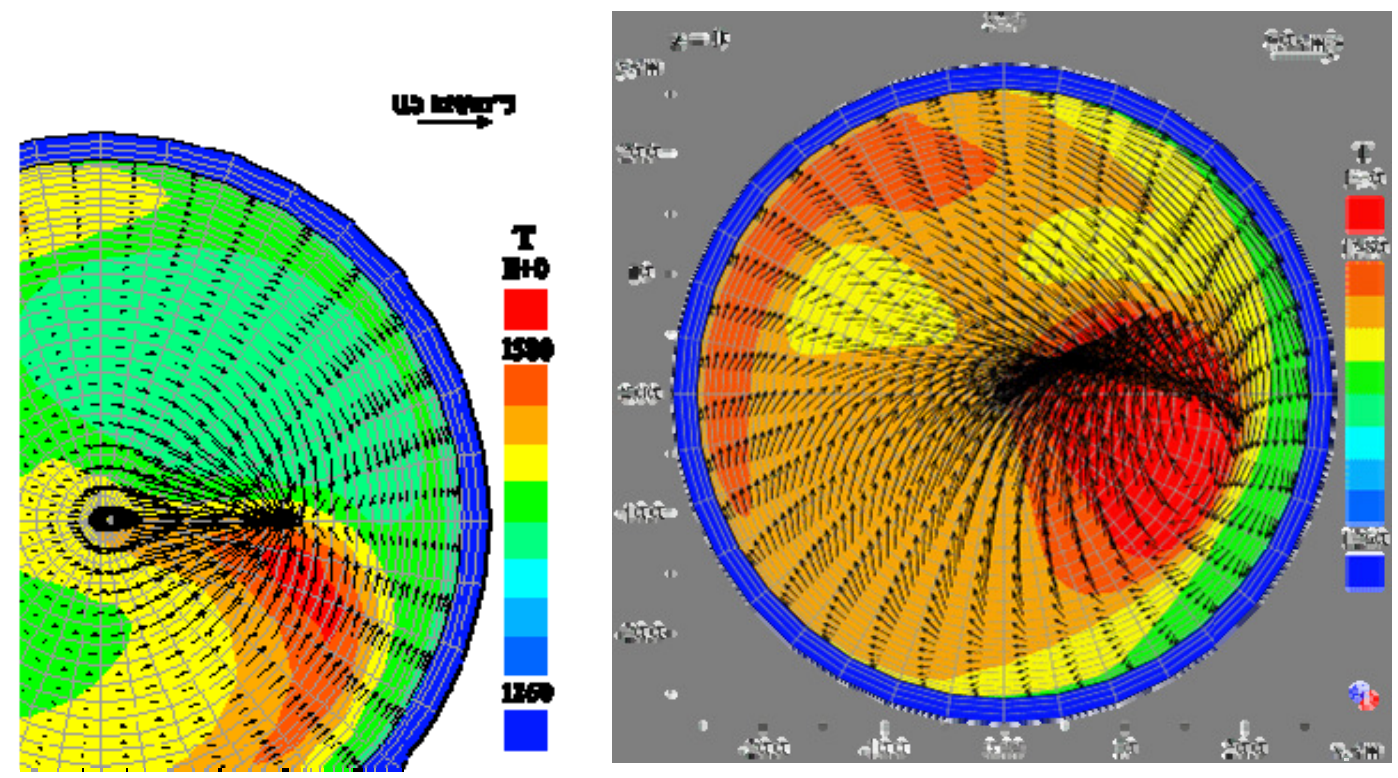

Figure 1. Left: the electromagnetic force distribution on the top of the liquid metal pool at a particular time moment $(\mathrm{t}=37 \mathrm{~s}$ from the start-up). Right: the velocity field at the same time moment on the top of the liquid metal pool.

The electric current distribution in the pool is very sensitive to the conditions at the side walls. If the conditions at the side wall of the remelted ingot are such that the side arc is not ignited, a significantly larger portion of the total current is forced through the liquid metal pool, generating more magnetic influence on the pool mixing and the solidification front (Figure 4, left). If the side arc is ignited, the current density in the metal pool and the magnetically induced mixing are reduced (Figure 4 , right). 


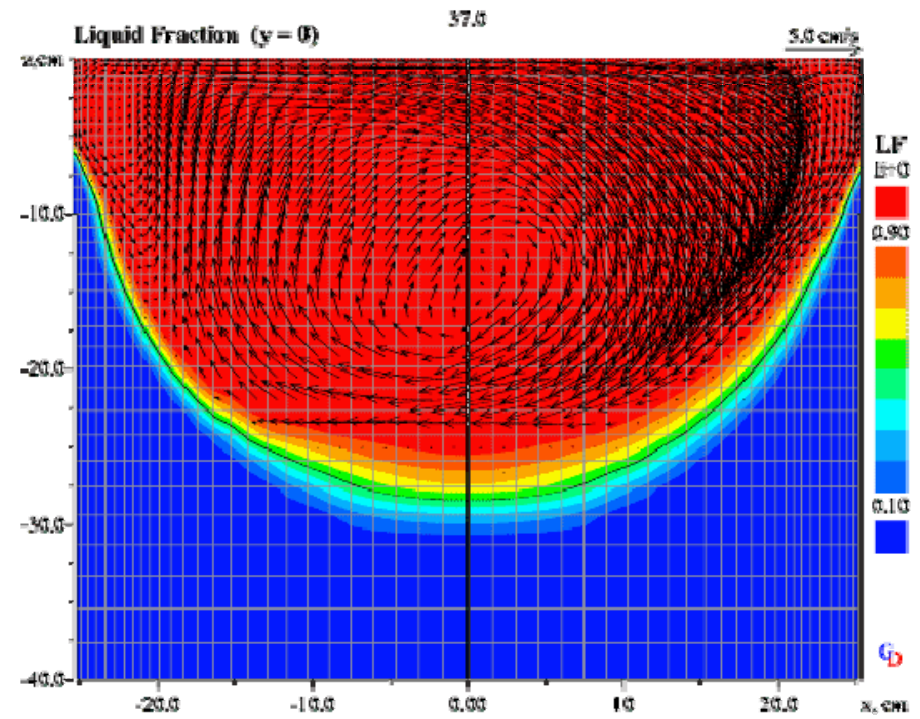

Figure 2. The flow structure and liquid fraction distribution in the liquid metal pool vertical cross section at the same time moment as in the Figure 1.
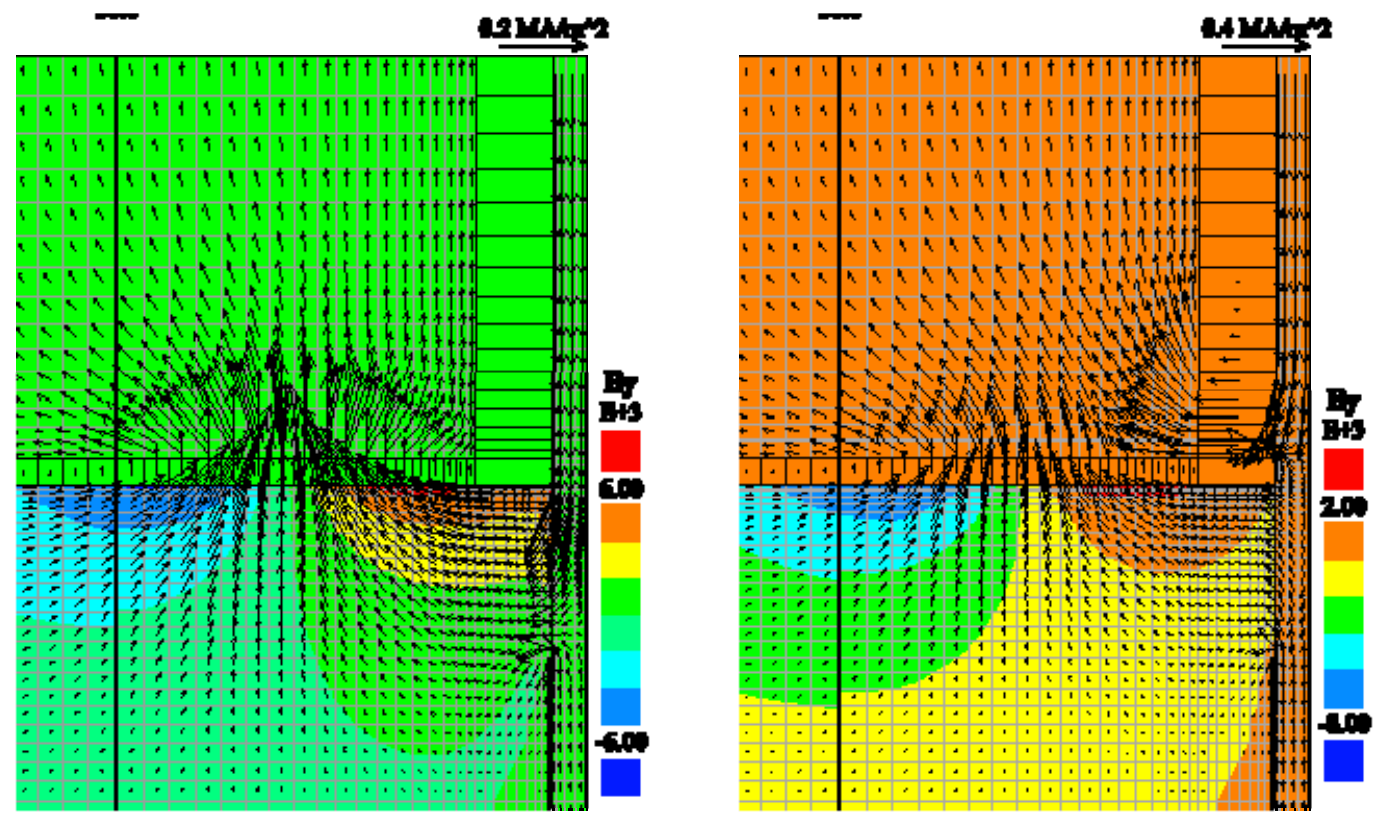

Figure 3. The electric current and the associated magnetic field distribution in the liquid metal pool, arc region, wall and the remelted ingot shown in a vertical cross section for two cases: left - no side arc, right - with the side arc.

The melting electrode film flow and the droplet detachment initiation are simulated separately by an axisymmetric transient model implemented in the code SPHINX [5,6]. The model predicts considerable Joule heating in addition to the arc radiation heating in the region of constricted droplet detachment. The electric current distribution at the initial and droplet detachment stages are shown in the Figure 4. The strong 'pinching' electromagnetic force appears at the later stage when a significant metal protrusion is developed via the intense electromagnetic mixing in the film. Time scales for the droplet creation via the electromagnetically driven film flow are found in the region of 100-300 ms depending on the film thickness. Local 'burn-outs' on the electrode surface are predicted at the droplet detachment (see Figures 5 and 6 at the various stages of the process and zoom-ins).

A unified 3-dimensional model is proposed for the electrode film and metal pool flow and heat transport in the future stage of this project. 

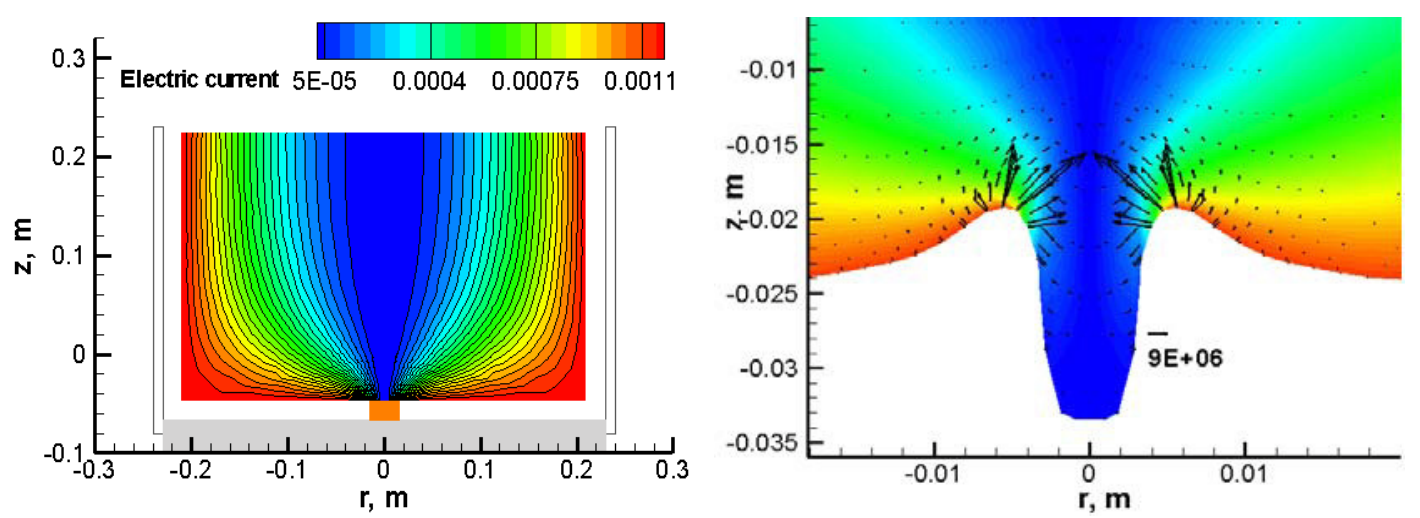

Figure 4. Left: the initial electric current and the associated magnetic field distribution in the remelted ingot (axisymmetric). Right: the current and electromagnetic force distribution at the later stage when a significant molten metal protrusion has developed.
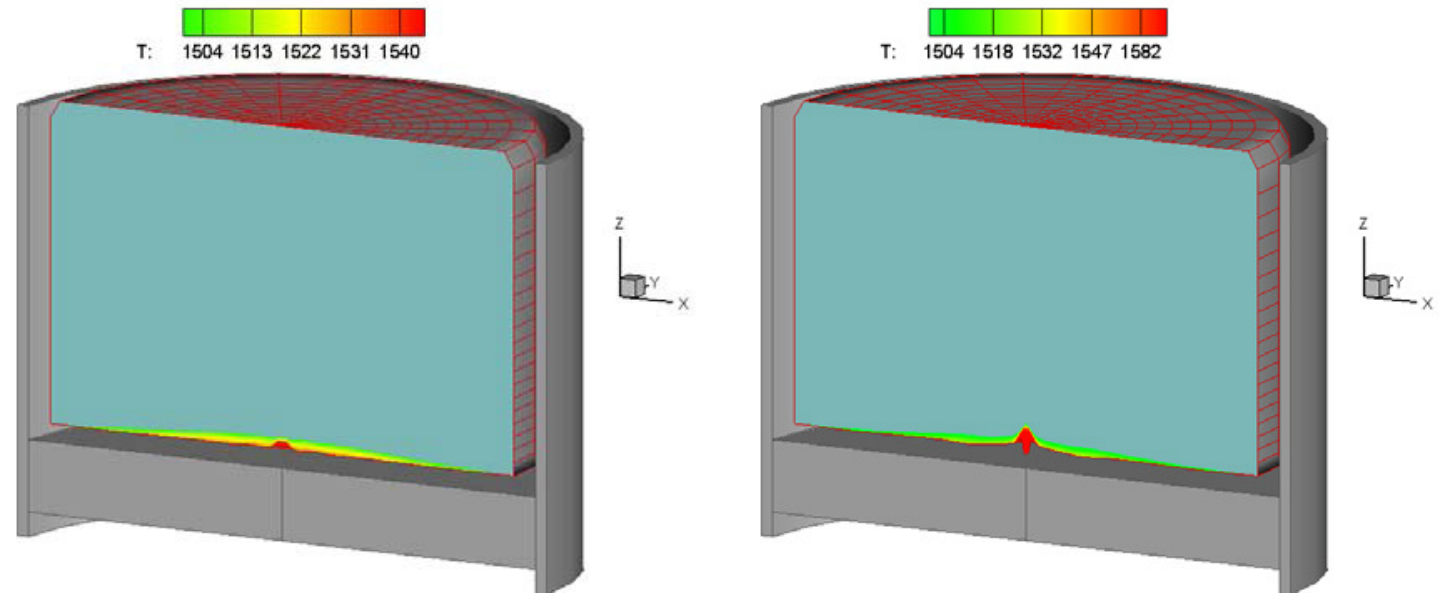

Figure 5. Left: the initial temperature distribution in the remelted ingot (axisymmetric). Right: the melt front and the liquid film shape at the later stage when a significant molten metal protrusion has developed.
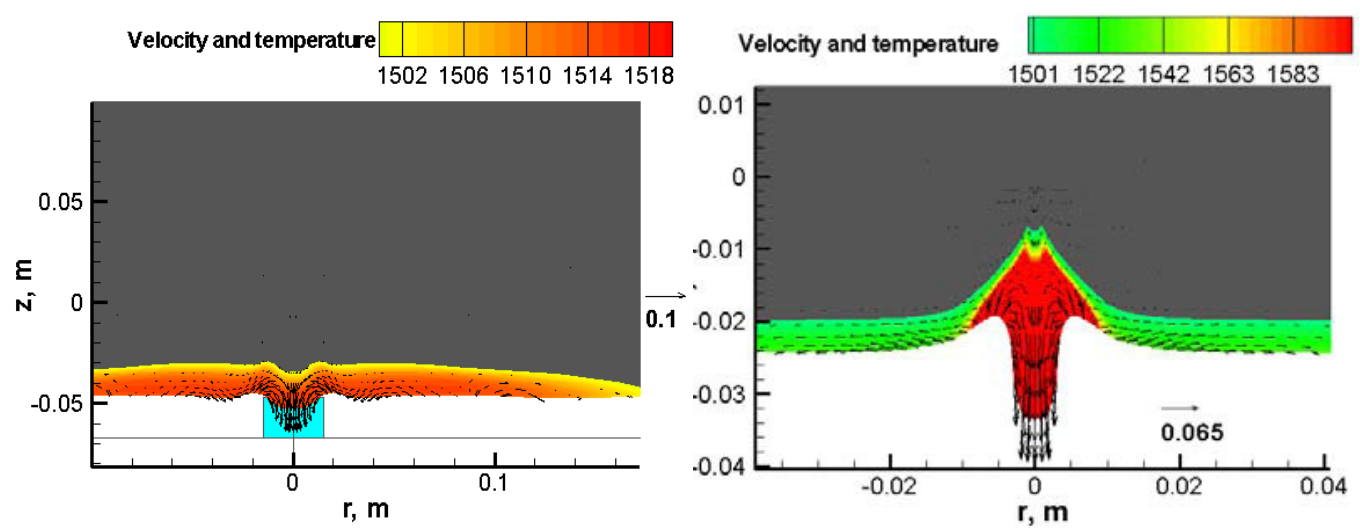

Figure 6. Left: the initial temperature and flow in the liquid metal at the bottom of the remelted ingot. Right: the melt front and the liquid film shape at the later stage when a significant molten metal protrusion has developed just before the droplet detachment.

\section{References}


[1] A. Jardy, S. Hans: Modelling Casting, Welding and Advanced Solidification Processes, C. A. Gandin (Ed.), TMS, Warrendale PA, 2006, pp. 953-960.

[2] D.M. Shevchenko and R.M. Ward: Proc. 2007 Int. Symp. Liquid Metal Processing and Casting, P. Lee (ed.), Nancy (France), 2007, pp. 25-30.

[3] www.physica.co.uk

[4] S. Bounds, G. Moran, K. Pericleous, M. Cross and T.N. Croft: Metall. Mater. Trans, 2000, 31B, pp. 515-527.

[5] V. Bojarevics, R.A. Harding, K. Pericleous and M. Wickins: Metall. Materials Trans., Vol. 35B, 2004, pp. 785-803.

[6] V. Bojarevics and K. Pericleous: ISIJ International, 2003, vol. 43, N 6, pp. 890-898.

\section{Authors}

Dr. Bojarevics, Valdis

School of Computing and Mathematics

University of Greenwich

30 Park Row

London SE10 9LS, UK

E-mail: v.bojarevics@gre.ac.uk

Dr. Djambazov, Georgi

School of Computing and Mathematics

University of Greenwich

30 Park Row

London SE10 9LS, UK

E-mail: g.djambazov@gre.ac.uk
Prof. Pericleous, Koulis

School of Computing and Mathematics

University of Greenwich

30 Park Row

London SE10 9LS, UK

E-mail: k.pericleous@gre.ac.uk 